\title{
LA INTERNACIONALIZACIÓN DE LA EDUCACIÓN SUPERIOR EN CHINA
}

\section{The Internationalization of Higher Education in China}

\author{
Xinran Liao * e Inmaculada Egido Gálvez *
}

\section{RESUMEN}

En el contexto de la globalización económica, la internacionalización de la educación superior se perfila como una tendencia que está modificando las agendas de reforma educativa de este nivel en todos los países. En el caso de China, las políticas adoptadas en los años pasados han tratado de ampliar el nivel de internacionalización de las instituciones superiores, pero aún son escasos los estudios destinados a profundizar en esta temática. Este artículo trata de contribuir al conocimiento de esta cuestión por medio de una revisión de la normativa, las estadísticas oficiales y las publicaciones existentes sobre el proceso de internacionalización de la educación superior en el país. El repaso a la situación actual de dicho proceso permite detectar algunos éxitos conseguidos durante los últimos años, pero pone también de manifiesto la existencia de problemas, por lo que cabe plantear algunas líneas de reflexión que pueden proponerse para el desarrollo futuro de las políticas de este ámbito.

PALABRAS CLAVE: Internacionalización, Educación superior, China.

\section{ABSTRACT}

In the context of economic globalization, the internationalization of higher education appears as a tendency that is modifying the educational reform agendas at this level in all countries. In the case of China, the policies adopted in recent years have been

\footnotetext{
${ }^{*}$ Universidad Complutense de Madrid (España).
} 
focused to expand the level of internationalization of higher institutions, but there are still few studies that analyse in depth this topic. This article seeks to contribute to the knowledge of this question through the review of regulations, official statistics and literature on the process of internationalization of higher education in this country. The review of the current situation of the process makes it possible to detect some successes in recent years, but also reveals the existence of problems, so it could raise some proposals for the future development of policies in this area.

KEY WORDS: Internationalization, Higher Education, China.

$* * * * * *$

\section{INTRODUCCIÓN}

Como es sabido, en el momento presente el conocimiento se ha convertido en el motor clave para el desarrollo de las naciones. En esta era de la globalización, la movilidad de la mercancía y los servicios ha perfilado un nuevo mercado internacional, que incluye también la educación superior (FARHAN, 2012: 3). Cualquier país, si quiere competir con los demás, depende de sus recursos de conocimiento y de su capacidad de transformarlos eficientemente en fuerzas productivas, por lo que la educación superior juega un papel clave en esta competición. La internacionalización, como nueva tendencia y como producto de la globalización, se ha integrado en el proceso de construcción y desarrollo de las instituciones superiores modernas.

Pero, concretamente ¿qué significa la internacionalización de la educación superior? Son muchos los investigadores $\mathrm{y}$ las organizaciones que han definido la internacionalización, con diferentes interpretaciones de este concepto. La Asociación Internacional de Universidades (International Association of Universities, IAU), ha adoptado en sus trabajos la definición de J. Knight, según la cual la internacionalización es un proceso destinado a integrar una dimensión intercultural o global en la finalidad, las funciones o la provisión de la educación postsecundaria (KNIGHT, 2003: 2). Otros autores aportan una definición más amplia, según la cual "este término tiende a ser utilizado para cualquier fenómeno supra-regional relativo a la educación superior [...] y/o a cualquier cuestión referida a la educación superior caracterizada por el mercado y la competición referida a escala global"1 (TEICHLER, 2004: 22-23).

Una de las facetas más visibles de la internacionalización es la movilidad de estudiantes, profesores e investigadores, pero el término incluye también otras dimensiones,

\footnotetext{
${ }^{1}$ La traducción al castellano de las citas textuales de fuentes originales en lengua inglesa y china que aparecen en el artículo ha sido realizada por las propias autoras.
} 
como la educación trasnacional, que se ofrece a través de campus universitarios trasladados a diferentes países, programas internacionales conjuntos, educación a distancia, etc. Además, forma parte también de este proceso la denominada "internacionalización en casa", expresión que se utiliza para hacer referencia a la incorporación de elementos interculturales e internacionales en el currículo, la enseñanza o la investigación que ayuda a los estudiantes a desarrollar sus habilidades internacionales sin dejar su país (OECD, 2004).

En los últimos años, la internacionalización de la educación superior ha cobrado un fuerte impulso. La IAU ha fundado un grupo destinado específicamente a la internacionalización de la educación superior y ha llevado a cabo encuestas e investigaciones a nivel mundial acerca de este tema, la última de ellas en el año 2014 (IAU, 2014). Además, investigadores de distintos países se han dedicado a esta cuestión, analizando las motivaciones que subyacen a este proceso, así como los efectos y los desafíos que el mismo provoca (AHMAD, 2012; CENTER FOR INTERNATIONALIZATION AND GLOBAL ENGAGEMENT, 2012; DE WIT, 2010; JONES y DE WIT, 2014).

La globalización ayuda a las instituciones académicas a salir de sus fronteras nacionales (DE WIT, 2011: 4) y ha hecho que la internacionalización sea una aspiración común a todas ellas, si bien el punto de partida de cada una no es el mismo, ya que "los estudiantes internacionales tienden a elegir los países e instituciones de mayor prestigio" (FARHAN, 2012: 11), atraídos por la mejor calidad de su formación y considerando la mayor demanda de sus titulaciones en el mercado laboral.

\section{LA INTERNACIONALIZACIÓN DE LA EDUCACIÓN SUPERIOR EN CHINA}

En el caso de China, existen muy pocos trabajos acerca del desarrollo de la internacionalización de la educación superior. Actualmente, China ha abierto la puerta a la educación internacional, colaborando con organizaciones y con instituciones superiores extranjeras. Esta tendencia podrá aumentar el nivel de internacionalización y la calidad educativa del país, si bien para ello debe afrontar diversas problemáticas, compartidas por otros países (ALTBACH, 2007: 15-16), como tratará de explicarse más adelante. Los investigadores chinos se han centrado mayoritariamente en la construcción de indicadores para evaluar la internacionalización, en resaltar los ejemplos y medidas más avanzadas desarrolladas en el extranjero o en analizar los efectos que supone la internacionalización de la educación superior en el país (LI, 2005; WANG y CHENG, 2007; ZHANG, 2012). Sin embargo, dada la corta historia del desarrollo de la internacionalización en China y la escasa trayectoria de las investigaciones al respecto, China aún queda relegada respecto a los países desarrollados en esta temática. Por esta razón, el objetivo de este artículo es contribuir a avanzar en la investigación sobre esta cuestión, realizando una revisión documental de la normativa, las estadísticas oficiales y las publicaciones existentes al 
respecto, con el fin de analizar la situación del país en lo que se refiere a la internacionalización de la enseñanza superior, detectar la problemática existente y realizar propuestas orientadas a la mejora.

Para facilitar la comprensión de este apartado, se presenta en primer lugar una breve descripción del marco contextual relativo a la educación superior en China. A continuación, se sintetizan las principales políticas orientadas a la internacionalización desarrolladas en el país y, finalmente, se expone la situación actual del proceso de internacionalización de la educación superior.

\subsection{El contexto de la educación superior en China}

Habitualmente, la historia de la educación superior moderna en China se divide en dos grandes periodos. El primero comienza en 1862, tras la fundación del Colegio Tongwen de Pekín y abarca hasta 1949, con el inicio de la nueva República Popular China (WANG y SONG, 2003: 47). Antes de la fundación de dicho Colegio no existía en China una educación superior moderna, sino la educación tradicional.

El segundo periodo comienza en 1949, con una fuerte influencia de la Unión Soviética, aunque el sistema cambió mucho a partir de 1970 (WANG y SONG, 2003: 48). Las políticas de "Reforma y Apertura" llevadas a cabo desde la década de 1990 dieron una nueva oportunidad de desarrollo a la educación superior y ampliaron mucho las comunicaciones y la cooperación con otros países, haciendo que la educación superior en China comenzara a buscar su propia ruta de desarrollo y de internacionalización.

Desde el punto de vista de la estructura, el actual sistema de educación superior en China es parecido al de Alemania, Japón y Estados Unidos, pero tiene características propias. Tras el examen de admisión para entrar en la universidad, los estudiantes eligen las instituciones en función de sus notas. Los estudios de Grado duran cuatro años y los de Máster duran habitualmente dos o tres cursos. En los últimos años, cada vez más estudiantes realizan el doctorado, respondiendo a las mayores demandas del mercado y de la sociedad. Normalmente se necesitan tres años o más para terminar los estudios de doctorado. Si es necesario se pueden seguir también estudios postdoctorales. Generalmente, para acceder al nivel superior desde el nivel previo de estudios es necesario superar exámenes, además de acreditar los méritos conseguidos, lo que supone la diferencia más significativa con los sistemas educativos alemán, japonés y norteamericano, mencionados anteriormente.

En el momento actual, el gobierno central y los gobiernos locales desarrollan políticas y ofrecen recursos para favorecer la internacionalización de la educación superior. En concreto, el Ministerio de Educación (MOE), el Ministerio de Relaciones Exteriores, el 
Consejo de Becas de China y el NOCFL (National Office for Teaching Chinese as Foreign Language) se encargan de orientar, supervisar y administrar todos los aspectos del desarrollo de la internacionalización de la educación superior. En diciembre de 2001, China se convirtió en miembro de la Organización Mundial del Comercio, lo que implica la apertura de sus fronteras a las instituciones de educación extranjera, ampliando las oportunidades para la internacionalización.

Como se ha mencionado, la internacionalización se encuentra estrechamente ligada a la globalización económica. En el caso de China, el rápido y sostenido desarrollo de la economía supone mayores oportunidades de comunicación y cooperación con otros países. Además, el progreso económico asegura mayores recursos para la educación y mayores oportunidades laborales, lo que estimula la mejora de la educación superior como principal ofertante de recursos humanos de alta calidad. En los últimos años, la economía china ha mantenido una tasa alta de crecimiento, de más del 7\% anual (NATIONAL BUREAU OF STATISTICS OF THE PEOPLE'S REPUBLIC OF CHINA, 2014), por lo que el país atrae la atención y la colaboración educativa internacional.

No obstante, junto a los factores económicos, China despierta también el interés internacional debido a su cultura. El país tiene una rica historia, de más de 5000 años, que atrae a millones de personas, incluidos estudiantes internacionales, que cada vez más eligen china para cursar sus estudios. Por otra parte, a medida que las comunicaciones mutuas entre China y el resto de los países se hacen más frecuentes y profundas, tanto la sociedad como los responsables de la educación superior se hacen más proclives a absorber ideas procedentes del exterior.

\subsection{Políticas orientadas a la internacionalización de la Educación Superior en China}

En los años pasados, distintos documentos gubernamentales han puesto de manifiesto la necesidad que tiene China de formar más personas con visión transcultural, capaces de conocer a fondo las normas internacionales y participar en asuntos internacionales (JIAN, 2011: 9). La educación superior precisamente es una esfera crítica para alcanzar esos objetivos, por lo que su internacionalización se ha convertido en un paso ineludible.

En concreto, el Ministerio de Educación ha desarrollado varios proyectos generales a nivel nacional, como el "Plan de Acción para la Promoción de la Educación 2003-2007" (MOE, 2004a) y el "Programa Nacional de Reforma y Desarrollo Educativo a Medio y Largo Plazo (2010-2020)" (MOE, 2010), proponiendo tanto ideas generales como acciones específicas para perfeccionar el nivel de internacionalización de la educación superior en China:

"La educación superior juega un papel substancial en formar personas capaces, avanzadas y especializadas, desarrollar la ciencia, la técnica y la cultura, y fomentar el proceso de modernización 
[...] es necesario estimular a las universidades a abrir sus asignaturas al mundo, apoyar su participación en las organizaciones internacionales de cooperación académica, así como en los planes científicos internacionales, y también establecer bases compartidas de I+D con las instituciones educativas de nivel superior y de investigación científica del extranjero.” (MOE, 2010)

En términos de las políticas desarrolladas, desde el año 2003 el gobierno chino ha aprobado los "Reglamentos de la Cooperación Educativa Chino-Extranjera" (MOE, 2003) y la normativa de desarrollo de la "Aplicación de Reglamentos de la Cooperación Educativa Chino-Extranjera' (MOE, 2004b). Sintetizando los contenidos de estas normas, las políticas perfiladas se centran en cuatro aspectos:

- Políticas destinadas a facilitar que tanto estudiantes como profesores chinos estudien en el extranjero. Entre ellas, se pueden diferenciar los estudios en el extranjero patrocinados por el gobierno, los estudios en el extranjero de auto-pago y las acciones destinadas a facilitar que los profesores enseñen en el extranjero.

- Políticas para los estudiantes extranjeros que van a estudiar a China, incluyendo las de admisión, enseñanza, titulación, administración, becas del alumnado extranjero, etc.

- Políticas para los estudiantes que regresan del extranjero e introducción de capital humano de ultramar.

- Políticas de cooperación educativa chino-extranjera.

Además, es importante destacar que en el año 2007 el Consejo de Estado aprobó el "Esquema del Undécimo Plan de 5 Años de China para el Desarrollo Educativo Nacional", en el que se propuso "fortalecer la cooperación y conversación internacional de la educación, y perfeccionar la apertura de la misma". Además, se planteó el "impulso para la educación cooperativa chino-extranjera" como uno de los cuatro deberes más importantes del Esquema (MOE, s.f.c). El contenido de este Plan, junto a los Reglamentos citados, impulsa la tendencia de incremento continuo de las acciones de cooperación educativa chino-extranjera, lo que supone un paso muy importante para la internacionalización de la educación superior.

\subsection{La situación actual del proceso de internacionalización de la educación superior en China}

Con el fin de conocer el alcance que el proceso de internacionalización de la educación superior en China tiene en estos momentos, se presentan a continuación una serie de informaciones e indicadores relativos a la presencia de estudiantes internacionales en el país, al número de estudiantes de origen chino que estudian en el extranjero, al intercambio de profesorado y a la cooperación educativa chino-extranjera. 


\subsubsection{Estudiantes Internacionales de educación superior en China}

En los últimos diez años, los estudiantes internacionales en China se han ampliado de 141.100 en 2005 hasta 377.000 en 2014, lo que supone un incremento del 267\% (gráfico 1).

\section{Gráfico 1. Estudiantes internacionales en China y estudiantes domésticos cursando estudios en el extranjero.}

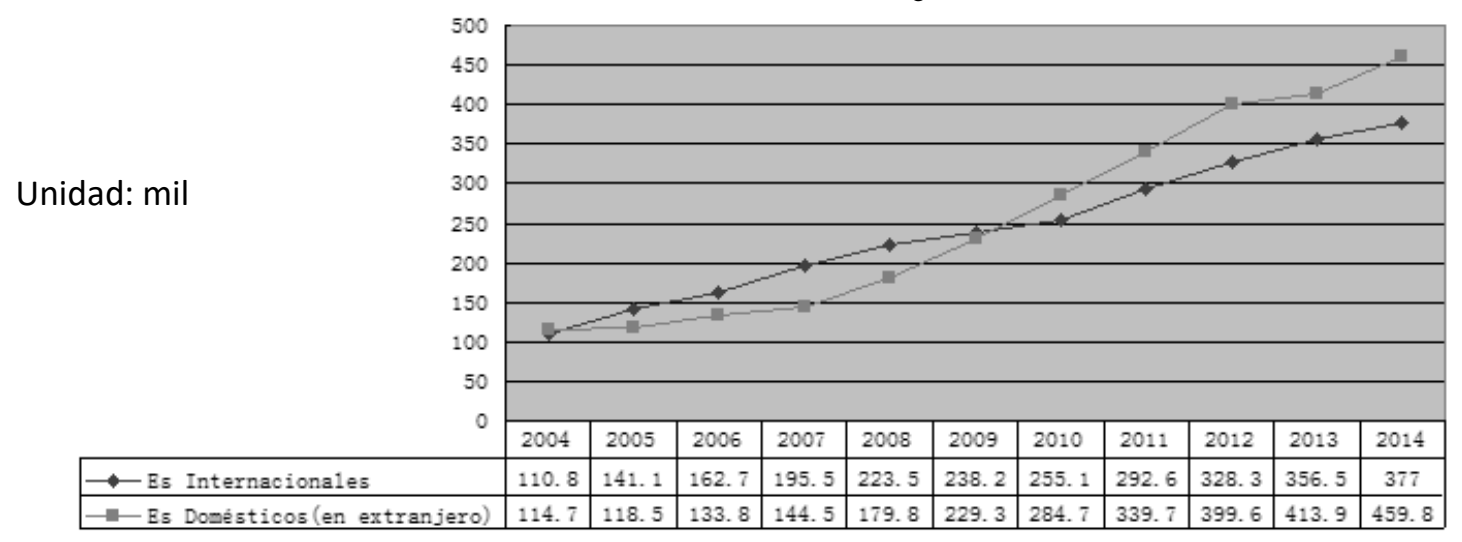

*Fuente: Elaboración propia a partir de las estadísticas de los años 2004-2014 del MOE

A causa de las influencias culturales y geopolíticas, la mayoría de dichos estudiantes proceden de los países asiáticos. En 2004, unos 85.000 estudiantes internacionales eran asiáticos, lo que constituía un 76,69\% del total (MOE, 2005). Este número ha alcanzado los 225.490 en 2014, pero ha bajado en términos porcentuales hasta ocupar el 59,8\% del total. Además, en los últimos años, el número de los estudiantes africanos y europeos ha experimentado un incremento mayor que el de los estudiantes de América y de Oceanía (MOE, 2015b). En 2004, los primeros 10 países de origen de los estudiantes que iban a China eran Corea, Japón, Estados Unidos, Vietnam, Indonesia, Tailandia, Rusia, Alemania, Francia y Nepal, por este orden (MOE, 2005). En 2014 estas cifras corresponden a Corea, Estados Unidos, Tailandia, Rusia, Japón, Indonesia, Vietnam, India, Kazajstán y Pakistán (MOE, 2015b). Corea es el país de origen de la mayor parte de los estudiantes internacionales que estudian en China.

Por lo que se refiere a la ubicación de estos estudiantes, en 2014 las 10 provincias o ciudades que recibieron más estudiantes internacionales eran Beijing, Shanghai, Tianjin, Jiangsu, Zhejiang, Guangdong, Liaoning, Shandong, Hubei y Heilongjiang. En un período largo, Beijing y Shanghai han sido las dos ciudades que recibieron más estudiantes internacionales en China. Aunque los porcentajes de estudiantes de las dos ciudades han disminuido un poco, se mantienen más o menos en torno al 40\% (MOE, 2015b). Esta distribución indica que los estudiantes internacionales se centran en las zonas litorales y desarrolladas o en los centros culturales, políticos y económicos, siendo muy poco el 
alumnado que acude a las zonas suroeste y noroeste del país, así como a otras provincias menos desarrolladas.

En relación con el tipo de estudios que cursan los estudiantes internacionales, las estadísticas del Ministerio de Educación indican que las carreras más populares son filología china, ciencia médica moderna y tradicional china, economía, administración e ingeniería. De ellas, los estudiantes de filología china agrupan al 60\% del total. La Tabla 1 ilustra esta cuestión, si bien debido a las limitaciones de los datos aportados por el Ministerio, sólo se muestran los estadísticos desde 2003 hasta 2010.

Tabla 1. Carreras cursadas por los estudiantes internacionales en China

\begin{tabular}{|l|l|l|l|}
\hline Carrera & 2003 & 2006 & 2010 \\
\hline Filología China & 75,99 & 70,59 & 62,53 \\
\hline Ciencia Médica Moderna & 3,86 & 8,13 & 9,51 \\
\hline Ciencia Médica Tradicional China & 5,38 & 4,38 & 4,14 \\
\hline Economía & 3,98 & 4,49 & 6,36 \\
\hline Administración & 1,99 & 3,66 & 5,63 \\
\hline Ingeniería & 3,47 & 3,57 & 5,71 \\
\hline
\end{tabular}

*Fuente: elaboración propia a partir de las estadísticas de los años 2003-2010 del MOE

\subsubsection{Estudiantes chinos de educación superior en el extranjero}

Estimulados por las políticas desarrolladas en los últimos años, cada vez más estudiantes chinos salen a estudiar al extranjero. Como muestra el gráfico 1, en el año 2004 un total de 114.700 estudiantes chinos estudiaban en el extranjero, cifra que en 2015 se ha incrementado a 523.700, lo que supone un aumento del $456 \%$ en una década (MOE, 2015a).

La mayoría de estos estudiantes estudian por su propia cuenta (90\% del total). Los principales destinos de estudio son Estados Unidos, Inglaterra, Australia y Canadá, países tradicionalmente poderosos en el ámbito de la educación superior, con un amplio mercado educativo. Además, el inglés, lengua oficial de estos países, es el idioma que casi todos los estudiantes chinos han estudiado a lo largo de su escolaridad. Este crecimiento vertiginoso del alumnado chino saliendo al extranjero a estudiar hace de China el mayor y más importante país de origen de estudiantes para el mercado educativo mundial. De acuerdo con los datos proporcionados por el Proyecto Atlas, los estudiantes chinos forman la 
principal parte de los estudiantes internacionales en Estados Unidos, Inglaterra, Australia, Canadá, Japón y Finlandia. Además, aunque no sean el grupo mayoritario, suponen una amplia proporción de los estudiantes internacionales en países como Alemania, Francia, España y Holanda (Institute of International Education, s.f.).

\subsubsection{Internacionalización del Profesorado}

En los últimos años, tanto el gobierno nacional como los locales han prestado una atención creciente a la atracción y la formación de personas con visión internacional, apoyando estas metas con políticas favorables y con financiación. Por esta razón, el intercambio internacional del profesorado chino es cada vez más frecuente. Tomando los Estados Unidos como ejemplo, durante el período de 2004 a 2015, los primeros cinco países que han emitido profesores a investigar y seguir estudios en Estados Unidos son China, India, Corea, Alemania y Japón, respectivamente. Además, mientras en los años mencionados la cantidad de profesorado en Estados Unidos procedente de Alemania e India se ha mantenido estable y la de Corea y Japón ha tenido una ligera tendencia de disminución, en el caso de China ha experimentado un crecimiento constante, pasando de 14.871 profesores en 2004 a 40.193 en 2014 (Gráfico 2).

\section{Gráfico 2. Profesorado extranjero cursando estudios en EEUU (2004-2015)}

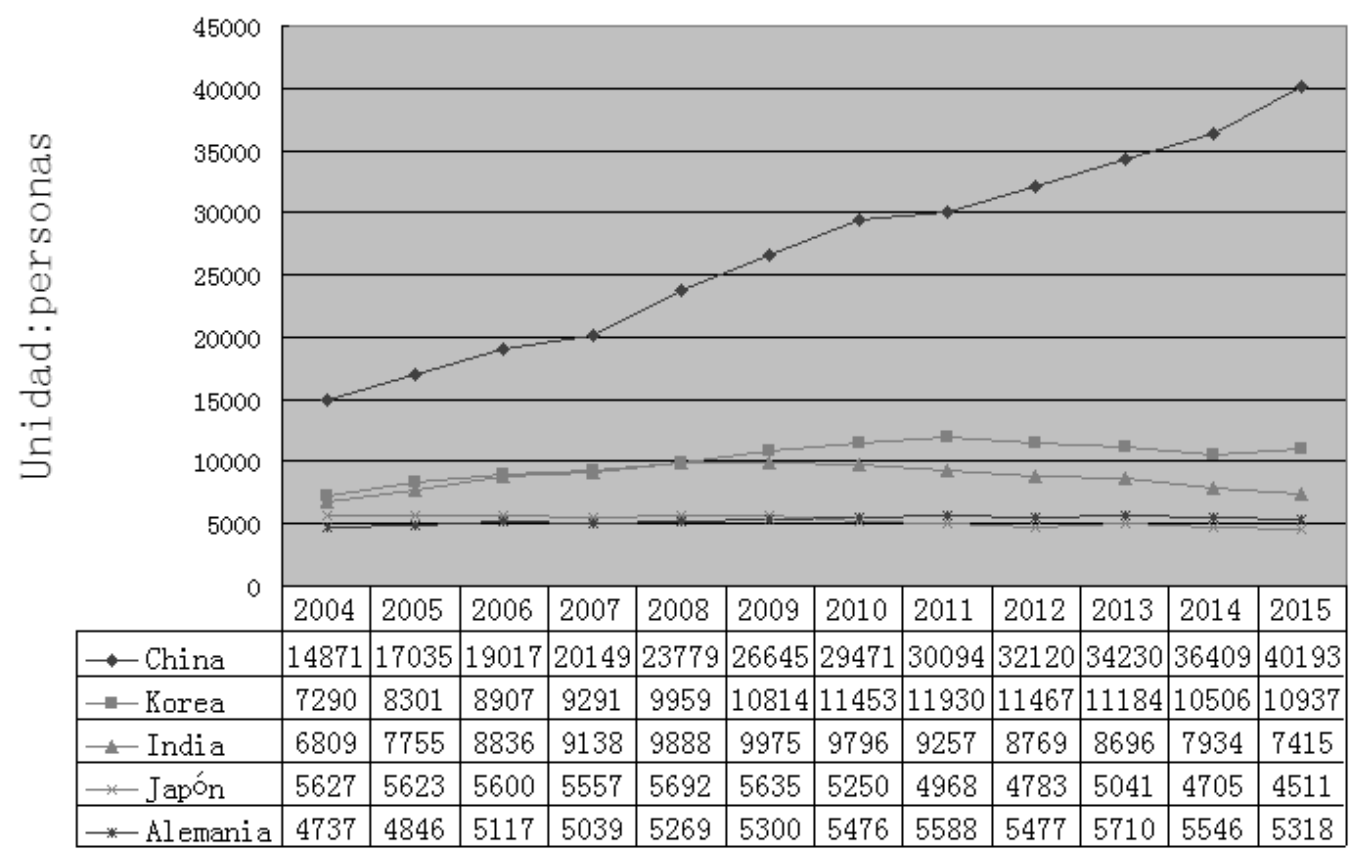

*Fuente: elaboración propia a partir de las estadísticas de los años de 2004-2015 del Institute of International Education (2015)

Por lo que se refiere a la atracción de profesorado extranjero al país, se han puesto en práctica diversas políticas favorables, como el "Programa de Reclutamiento de Expertos 
Globales". Ello ha hecho que la cantidad de docentes extranjeros haya aumentado en la última década, siendo actualmente de 6.269 la cifra de profesores extranjeros de doctorado, 4.510 los de máster y 4.596 los de licenciatura (MOE, s.f.b).

\subsubsection{Cooperación educativa chino-extranjera}

La cooperación educativa chino-extranjera se concreta tanto en las acciones desarrolladas dentro del territorio chino como en las llevadas a cabo en el extranjero (LU, KANG y YAN, 2013: 75). En el primer caso, este tipo de educación superior posibilita a los estudiantes tener una experiencia internacional sin salir de su propio país (CHINESEFOREIGN COOPERATION IN RUNNING SCHOOLS, s.f.). En 2003 existían 712 programas de cooperación educativa chino-extranjera en China, sirviendo a unas 100.000 personas (LI y WANG, 2009: 97). Según el Ministerio de Educación, en este momento hay unos 450.000 alumnos estudiando en las instituciones cooperativas chino-extranjeras y son más de 1.500.000 los graduados de estas instituciones. Hasta diciembre de 2015, los programas cooperativos aprobados por el ministerio ascienden a 894 (MOE, s.f.a).

La cooperación educativa en China se ha desarrollado fundamentalmente desde Inglaterra, Estados Unidos y Australia, que han establecido en el país centros universitarios. Además, debido a su posición geográfica, Rusia también ha establecido muchas instituciones y programas en China, si bien se centran sobre todo en la región noreste del país, especialmente en Heilongjiang, provincia limítrofe con el territorio ruso. En cuanto a las carreras ofertadas en territorio chino por las universidades extranjeras, su número es superior a 1.200, si bien las titulaciones de las áreas de ingeniería y economía agrupan el $40 \%$ de las mismas, seguidas por administración de empresas e informática, que suponen aproximadamente un 30\% del total (Gráfico 3).

\section{Gráfico 3. Distribución de carreras de la cooperación educativa chino-extranjera}

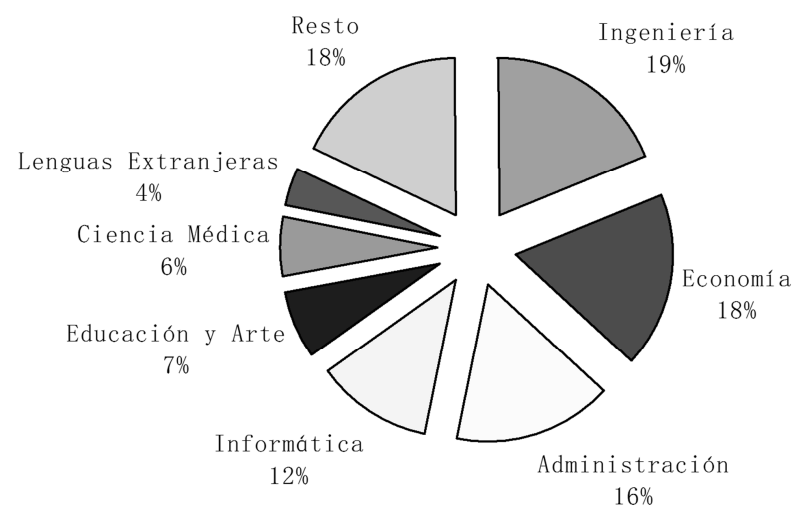

*Fuente: elaboración propia a partir de las estadísticas del año 2014-2015 de MOE 
Por lo que se refiere a la cooperación educativa de China en el extranjero, en el año 2003 unas 20 universidades chinas se establecieron en otros países, centrándose en Singapur, Malasia, Japón e Inglaterra, con carreras principalmente de filología china y medicina tradicional china (LI y LIU, 2008: 45). Las universidades chinas también han comenzado a salir al extranjero, siguiendo la tendencia de desarrollo de la educación superior en el mundo. Por ejemplo, la Universidad de Xiamen ha establecido su campus en Malasia y ha empezado a admitir nuevos alumnos desde el otoño de 2015 (FMPRC, 2016).

\section{PROBLEMÁTICA DE LA INTERNACIONALIZACIÓN DE LA EDUCACIÓN SUPERIOR EN CHINA}

Teniendo en cuenta lo expuesto hasta el momento, se puede observar que en los años pasados el esfuerzo de China para avanzar en la internacionalización de la educación superior ha sido relevante. La colaboración y los intercambios educativos y culturales entre las instituciones domésticas e internacionales son cada día más frecuentes, gracias a las políticas gubernamentales y a los incentivos de intercambio internacional para el alumnado y el profesorado (HONG, 2013: 31). Desde la perspectiva del efecto práctico de estos esfuerzos, la comunicación y el contacto con diferentes culturas pueden contribuir a formar la visión y el pensamiento internacional del profesorado y del alumnado y a fomentar la comparación y la reflexión en perspectiva internacional (DU y SUN, 2012: 64-65). Sin embargo, el proceso de internacionalización de la educación superior en China no está exento de problemas, como tratará de ponerse de manifiesto a continuación.

\section{$\underline{\text { 3.1. Idea superficial de la internacionalización de la educación superior }}$}

Tras más de dos décadas de desarrollo, se puede decir que la internacionalización de la educación superior en China ha avanzado progresivamente hacia un planteamiento relativamente más profundo y adecuado para el país, evolucionando desde la pura imitación que se llevó a cabo en el pasado a posiciones más maduras. No obstante, este planteamiento se encuentra aún en un nivel bajo, dado que muchos investigadores, administradores, políticos, profesores y estudiantes no entienden este concepto con profundidad. Muchos de ellos solamente consideran la internacionalización como trabajar, investigar, estudiar con los extranjeros, sin considerar que el núcleo y la esencia de la internacionalización es la formación de personas con una alta capacidad de visión y conciencia de la internacionalización, la globalización y la problemática socio-cultural del planeta (VÁZQUEZ COVARRUBIAS, 2006: 93). En realidad, muchas instituciones tienen sus propios colegios internacionales $\mathrm{u}$ organizaciones administrativas para la internacionalización, pero en la práctica estos se limitan a admitir y a administrar a los estudiantes internacionales, sin una idea amplia sobre qué es internacionalización. La internacionalización de la educación superior no sólo significa admitir estudiantes internacionales, abrir unas clases transculturales, participar en las comunicaciones 
académicas internacionales o estudios cooperativos, ni tampoco únicamente salir de su propio país a estudiar en el extranjero. La internacionalización necesita una transferencia amplia y profunda de ideas, la introducción de las experiencias de otros países y al mismo tiempo la difusión al mundo de la cultura y la educación superior china.

\subsection{Disparidad entre regiones, instituciones y carreras}

El desarrollo de la internacionalización de la educación superior en China presenta fuertes discrepancias en lo que se refiere a su situación regional, institucional y por tipo de estudios. Así, como se ha reflejado en el apartado anterior, hay una desigualdad muy significativa desde el punto de vista geográfico, dado que la gran mayoría de estudiantes internacionales se sitúa en las regiones costeras, fronterizas o las más desarrolladas económica y culturalmente, como Shanghai o Beijing, así como en las ciudades que actúan como centros de atracción en lo que se refiere a la cultura y la educación y que se caracterizan por disponer de una industria educativa avanzada, como por ejemplo Wuhan y Chengdu, capitales de la provincia Hubei y Sichuan respectivamente. En comparación con dichas regiones, las instituciones de las zonas de noroeste y suroeste del país reciben muchos menos estudiantes internacionales y los que acuden a ellas suelen ser los de los países vecinos, como Tailandia, Vietnam o Camboya, que tienen un nivel de formación inferior al de los estudiantes occidentales.

Por tipo de carreras, como se ha mencionado, unas pocas de ellas reciben a la mayoría de los estudiantes internacionales. Especialmente destaca a este respecto la carrera de filología china, que recibe a más del $60 \%$ de los estudiantes internacionales. Ello implica que otros estudios no resultan atractivos para los estudiantes de fuera, por lo que urge el desarrollo de la internacionalización de los mismos.

\subsection{Problemas de la internacionalización en casa}

En la actualidad, China "se encuentra entre los países con mayor incremento de estudiantes que cursan sus carreras en instituciones internacionales dentro de su propio país" (WANG, ALTYNBEKOVA y LIU, 2014: 60), lo que guarda una estrecha relación con la rapidez del desarrollo económico y la gran demanda de personas altamente cualificadas. La internacionalización de los estudiantes domésticos significa crear oportunidades para quienes no pueden estudiar en el extranjero y ofrece la experiencia de contactos multiculturales. Sin embargo, en el caso de China este tipo de internacionalización plantea diferentes problemas (JON, 2013; PARSONS, 2010; XU, 2015):

- Los estudiantes tienen un concepto transcultural muy superficial. Muchos de ellos consideran a los estudiantes internacionales como "los occidentales", cuando, como se ha comentado anteriormente, en realidad prácticamente la mitad de estos 
estudiantes no vienen de occidente, sino que proceden de países asiáticos. Además, por la falta de fluidez del idioma para comunicarse, muchos estudiantes domésticos pierden la oportunidad de mantener contactos transculturales.

- Falta de oportunidades de interacción con los estudiantes internacionales. Un gran número de alumnos internacionales recibe sus clases separado del alumnado chino, debido a que muchos de ellos van a China a estudiar solamente su idioma. Además, para facilitar la administración, los estudiantes internacionales viven separados de los estudiantes domésticos, lo que dificulta la potencial interacción entre ambos grupos.

- Discrepancias culturales. El contacto con la diversidad cultural es esencial para la internacionalización, pero la dificultad para abrirse a otras culturas es un obstáculo para los estudiantes chinos, que debe superarse para lograr una verdadera interacción con las personas procedentes de otros países.

\subsection{Problemas derivados del "préstamo político" y de las "políticas reactivas"}

El análisis del proceso de internacionalización de la educación superior en China permite observar que muchas instituciones y gobiernos locales, e incluso a veces el gobierno central, establecen políticas directamente extraídas de las llamadas experiencias de excelencia, en las cuales el modelo adoptado procede de países extranjeros, habitualmente occidentales. Si los países en desarrollo solamente reforman sus sistemas por "préstamo político" (RAFFE, 2011), existe el peligro de que la internacionalización "sea una pura versión moderna de imperialismo cultural orientando a la creación de una sociedad universal y occidental, al fin y al cabo" (SHARMA, 2012: 1). De hecho, como se ha alertado, "la educación occidental ya ha reemplazado en realidad las formas indígenas de educación en el mundo tras ese proceso de internacionalización” (ORR, 1999: 166).

Además de lo anterior, una característica de los países en desarrollo es que adoptan políticas de carácter reactivo, en gran medida como una respuesta pasiva a los desafíos de la globalización (ALTBACH, 2003: 119). A este respecto, la mayoría de los artículos, tesis o estudios desarrollados acerca de la internacionalización de la educación superior en China llevan por título expresiones como "desafíos" o "enfrentarse." Este tipo de pensamiento, como "estímulo-reacción" influye en las políticas educativas y se refleja en las estrategias de recursos humanos, modificación de estructura, etc. La falta de iniciativa propia en la internacionalización de la educación superior significa no sólo que ésta está controlada por los países poderosos, sino que existe más dependencia que relación equilibrada entre distintas partes del mundo. 


\section{A MODO DE CONCLUSIÓN: ALGUNAS PROPUESTAS PARA EL} DESARROLLO DE LA INTERNACIONALIZACIÓN DE LA EDUCACIÓN SUPERIOR EN CHINA

La educación superior en China se encuentra ya en la órbita de la globalización y la internacionalización, si bien los problemas detectados en el proceso seguido hasta el momento hacen necesaria una reflexión que oriente una reforma sistemática de cara al futuro. Esa reforma debe planificarse a largo plazo e implica una gran complejidad, ya que debe tener un planteamiento global, que abarque la totalidad de la realidad educativa, para no caer en la superficialidad. No obstante, partiendo de lo expuesto en las páginas anteriores, se perfilan a continuación algunas propuestas de cambio que deberían ser consideradas de cara al futuro en China para que el proceso de internacionalización redunde realmente en una mejora de la calidad de la educación en el país.

Sin lugar a dudas, el cambio más necesario en China es el que se deriva de una mejor y más profunda comprensión del proceso de internacionalización de la educación superior y de su alcance. Más allá de las políticas de "salir" y "traer" que se han llevado a cabo en el terreno económico en China (YUEH, 2012: 2, 10), en el ámbito educativo, y especialmente en la educación superior, son necesarias ideas abiertas. El concepto de educación de las instituciones superiores del país tiene que mezclarse con el mundo, especialmente en las universidades que aún tienen ideas atrasadas y adolecen de falta de innovación. Estas universidades deben abrirse al mundo y seguir con atención las tendencias de desarrollo de la educación superior en otros contextos. Además, deben utilizar los indicadores internacionales para evaluar los logros educativos en la internacionalización.

Junto a lo anterior, China necesita avanzar en la superación de las disparidades regionales en la internacionalización de la educación superior antes mencionadas que hacen que casi la mitad de los estudiantes extranjeros en el país se concentren en solo dos ciudades, Beijing y Shanghai. Sin duda, las razones que causan estas discrepancias son complejas y tienen que ver con el desarrollo económico, cultural y social de las distintas regiones del país. Sin embargo, "entre las zonas menos desarrolladas de China existen algunas, como Xinjiang, Guangxi o Yunnan, que han atraído a numerosos estudiantes internacionales, a pesar de su peor situación de partida" (GU, 2010: 4). Ello implica que el gobierno central y los gobiernos locales deben establecer políticas favorables al desarrollo de la internacionalización, como becas o fomento de intercambios y comunicaciones en las regiones menos desarrolladas, para que aumente la atracción de estudiantes internacionales hacia las mismas. Pero también es necesario que las instituciones de estas regiones lleven a cabo acciones para aprovechar en mayor medida sus propios recursos culturales y sociales y desarrollen currículos adaptados a las demandas de estos estudiantes. Los buenos resultados obtenidos hasta el momento por las políticas preferenciales para las zonas 
mediterráneas subdesarrolladas llevadas a cabo por el gobierno (MOE, 2010), indican que es posible acelerar el proceso de internacionalización de la educación superior en estas regiones y reducir las desigualdades de carácter regional.

Por lo que se refiere a las discrepancias entre carreras, resulta previsible que el rápido desarrollo económico de China y su amplio mercado nacional hagan aumentar en el futuro la cantidad de alumnado extranjero interesado en cursar sus estudios en China. Ello producirá cambios en la situación actual, en la que, como se indicaba en la tabla 1, la mayoría de los estudiantes extranjeros acuden a cursar estudios de filología, "pero para ello se necesitan acciones destinadas al refuerzo de la internacionalización de nuevos tipos de estudios" (ZHAO, 2014: 22).

En relación con los estudiantes de educación superior de China, resulta evidente la necesidad de reforzar el aprendizaje de idiomas, especialmente el inglés. Los análisis realizados indican que los estudiantes chinos tienen ganas de comunicarse y contactar con los estudiantes internacionales, pero tienen como principal obstáculo el dominio de otro idioma (WANG, ALTYNBEKOVA y LIU, 2014: 64). Pero, además del idioma, es necesario reforzar el aprendizaje transcultural, con el fin de promover el respeto y entendimiento mutuo entre culturas. A este respecto, es esencial que las instituciones promuevan la interacción entre los estudiantes internacionales y los domésticos, favoreciendo su presencia conjunta en las mismas clases. Los entornos de aula ofrecen circunstancias muy favorables para la interacción social (OTTEN, 2000: 18) y permiten promover el intercambio de ideas y el desarrollo de capacidades transculturales.

No obstante, y sin olvidar los factores mencionados, lo fundamental en el proceso de internacionalización de la educación superior en China es que el país diseñe su propia estrategia sistemática de desarrollo, ya que las políticas reactivas a las que antes se ha hecho referencia no tienen validez para alcanzar la meta de fortalecer la educación superior. A la hora de crear esa estrategia en el país, es necesario considerar dos aspectos básicos. En primer lugar, el estatus en el que se sitúa la educación superior en China en relación a otros países y, en segundo lugar, las metas generales a las que se encamina el proceso de internacionalización de la educación superior.

Respecto al primer factor, es obvio que hoy en día, y a pesar del rápido desarrollo experimentado en los últimos años, la educación superior en China no se encuentra entre las mejores del mundo, ni puede posicionarse al nivel de países como Estados Unidos, Alemania o Japón. Así, de acuerdo con el Academic Ranking of World Universities (SHANGHAI JIAO TONG UNIVERSITY, 2015) en el año 2015 ninguna de las universidades de China se encontraba entre las 100 primeras del mundo. Por su parte, otras clasificaciones también ampliamente conocidas, como los World University Rankings (TIMES HIGHER EDUCATION, 2016) y QS World University Rankings 
(QUACQUARELLI SYMONDS, 2016) sitúan a la primera universidad china, la de Pekín, en torno al número 40 del top mundial. China sigue siendo una nación de influencia limitada en el sistema mundial del conocimiento, carente de poder de discurso en el proceso de internacionalización de la educación superior (ZHA,2008). En parte, ello es debido a que comenzó más tarde que los demás países el proceso de modernización económica, política y cultural, por lo que no cuenta con un sistema universitario equiparable al de los países mejor posicionados en el mercado mundial de la educación superior.

Respecto a las metas a las que se dirige el desarrollo de la internacionalización, China no debe aspirar únicamente a convertirse en un país fuerte en la oferta y la demanda dentro del "mercado global" de la educación superior, adoptando modelos procedentes de otros entornos culturales, sino que debe considerar la contribución que este nivel de enseñanza deber suponer para el desarrollo futuro del país, más allá de su mero papel como bien de consumo.

El papel de China puede ser muy importante para otros países en desarrollo: su participación en la construcción de los estándares educativos internacionales resulta necesaria, con el fin de que la internacionalización de la educación superior en el futuro no sea favorable sólo para los países desarrollados, sino también para aquellos que están en vías de desarrollo. El proceso de internacionalización de la educación no puede convertirse "en un mecanismo destinado a occidentalizar el mundo entero, sino que debe tener en consideración las diferentes culturas y sociedades" (SHARMA, 2012: 4), utilizando distintas aproximaciones y formas de enseñanza. Sin abandonar lo que puede aprenderse sobre los logros conseguidos en los países que cuentan con una educación más avanzada, es necesario prestar atención a la propia cultura, a los propios conceptos y tradiciones educativas Ello implica que, desde las políticas educativas, resulta imprescindible considerar la perspectiva local y nacional. Como indicó Altbach (2003), la educación superior del tercer mundo sólo tiene un camino de desarrollo, el camino endógeno, buscando un enfoque de desarrollo autónomo. Es necesario tener en cuenta que la internacionalización de la educación superior es un "arma de doble filo", por lo que es muy importante mantener el equilibrio entre las necesidades nacionales y la tendencia internacional (ALTBACH, 2003: 121). A pesar de encontrarnos en un entorno internacionalizado, la educación superior sigue siendo una responsabilidad clave de cada uno de los países, que deben asegurarse que sus intereses nacionales son atendidos y garantizar su calidad.

\section{REFERENCIAS BIBLIOGRÁFICAS}

AHMAD, S. (2012): Internationalization of Higher Education: A Tool for Sustainable Development, OIDA International Journal of Sustainable Development, 4, pp.79-90. 
ALTBACH, P. G. (2003): Knowledge and Education as International Commodities: The Collapse of the Common Good, Jiangsu Higher Education, 4, pp. 119-121.

ALTBACH, P. G. (2007): Chinese Higher Education in an Open-Door Era, International Educator (https://www.highbeam.com/doc/1P3-1308429811.html), consultado el 10 de abril de 2016.

CENTER FOR INTERNATIONALIZATION AND GLOBAL ENGAGEMENT (2012): Mapping Internationalization on U.S. Campuses: 2012 Edition (http://www.acenet.edu/news-room/Documents/Mapping-InternationalizationonUS-Campuses-2012-full.pdf), consultado el 1 de mayo de 2016.

CHINESE-FOREIGN COOPERATION IN RUNNING SCHOOLS (2005): Situación de la Educación Cooperativa Chino-Extranjera desde la aplicación del esquema de implementación de planteamiento de educación (http://www.crs.jsj.edu.cn/index.php/default/news/index/80), consultado el 4 de abril de 2016.

DE WIT, H. (2011): Globalisation and internationalisation of higher education, RUSC: Revista de Universidad y Sociedad del Conocimiento, 8, pp. 241-248.

DU, Y. y SUN, L. (2012): On the Internationalization of Adult Higher Education and the PSB Academy in Singapore, Journal of Hebei Normal University, 14, pp. 61-66.

FMPRC (Embassy of the People's Republic of China in Malaysia) (2016): Ambassador Huang Huikang attended the opening ceremony of the first batch of freshmen of Xiamen University Malaysia Campus (http://www.fmprc.gov.cn/ce/cemy/chn/sgxw/t1342497.htm), consultado el 8 de abril de 2016.

FARHAN, B. Y. (2012): The Impact of Globalization on Higher Education: Discussion and Critique (http://ssrn.com/abstract=2139138), consultado el 7 de abril de 2016.

GU, J. (2010): An analysis of developmental differences of higher education internationalization in China, Journal of Zhejiang Education Institute, 6, pp. 1-6.

HONG, L. (2013): On the Overseas Chinese Students in the Context of the Educational Globalization, Journal of Hebei Normal University, 15, pp. 29-33.

INSTITUTE OF INTERNATIONAL EDUCATION (2015): “Top 25 Places of Origin of International Scholars, 2013/14 - 2014/15” Open Doors Report on International Educational Exchange (http://www.iie.org/Research-and-Publications/OpenDoors/Data/International-Scholars/Leading-Places-of-Origin/2013-15), consultado el 3 de abril de 2016.

INSTITUTE OF INTERNATIONAL EDUCATION (s.f.): China's Students Overseas (http://www.iie.org/Services/Project-Atlas/China/Chinas-Students-Overseas), consultado el 3 de abril de 2016.

IAU (INTERNATIONAL ASSOCIATION OF UNIVERSITIES) (2014): IAU 4th Global Survey (2014) Internationalization of Higher Education - Growing expectations, fundamental values (http://www.iau-aiu.net/content/iau-global-surveys), consultado el 18 de abril de 2016.

JIAN, Y. (2011): Thoughts About the Actuality of Intenationalization of Higher Education in China, Guide of Sci-tech Magazine, 2, pp. 9-20.

JONES, E. y DE WIT, H. (2014): Globalized internationalization: Implications for Policy and Practice, Institute of International Education IIE Networker Magazine, pp. 2829. 
KNIGHT, J. (2003): Updated definition of internationalization, International Higher Education, 33, pp. 2-3.

LI, S. (2005): The Evaluation Index System of Internationalization of Universities, Journal of South China Normal University (Social Science Edition), 6, pp. 113-116.

LI, J. y LIU, Z. (2008): New Exploration on the Development Strategy of "Going Out" of Chinese-foreign Cooperation in Running Higher Education, Educational Research, 1, pp. 43-47.

LI, S. y WANG, Z. (2009): Thirty Years' Transnational Education in China-Based on 11 Provinces and Municipalities, Journal of South China Normal University (Social Science Edition), 2, pp.96-99.

LU, G., KANG, H. y YAN, N. (2013): On the Current Situation, Problems and Development Countermeasures of Sino-Foreign Cooperative Education, Research in Higher Education of Engineering, 4, pp. 75-80.

MOE (2003): Order No.372 of the State Council of the People's Republic of China (http://www.moe.gov.cn/jyb_xxgk/gk_gbgg/moe_0/moe_9/moe_35/tnull_96.html), consultado el 20 de abril de 2016.

MOE (2004a): Action Plan for the Revitalization of Education of 2003-2007 (http://www.moe.gov.cn/jyb_sjzl/moe_177/201003/t20100304_2488.html), consultado el 12 de marzo de 2016.

MOE (2004b): Order No. 20 of the Ministry of Education of the People's Republic of China (http://www.moe.gov.cn/jyb_xxgk/gk_gbgg/moe_0/moe_1/moe_162/tnull_2544.ht $\mathrm{ml})$, consultado el 20 de marzo de 2016.

MOE (2005): 2004 Statistical Yearbook of the International Students in China (http://www.moe.edu.cn/publicfiles/business/htmlfiles/moe/moe_850/201001/xxgk_ 77817.html), consultado el 20 de marzo de 2016.

MOE (2010): Outline of the National Medium and Long Term Educational Reform and Development Plan (2010-2020) (http://www.moe.edu.cn/publicfiles/business/htmlfiles/moe/moe_838/201008/93704 .html), consultado el 20 de marzo de 2016.

MOE (2015a): Situación de los estudiantes domésticos estudiando en el extranjero (http://www.moe.gov.cn/jyb_xwfb/gzdt_gzdt/s5987/201603/t20160316_233837.ht $\mathrm{ml}$ ), consultado el 20 de marzo de 2016.

MOE (2015b): Statistics of 2014 of the International Students in China (http://www.moe.gov.cn/jyb_xwfb/gzdt_gzdt/s5987/201503/t20150318_186395.ht $\mathrm{ml}$ ), consultado el 21 de marzo de 2016.

MOE (s.f.a): Lista de Instituciones y Programas Cooperativos Chino/Extranjeros (http://www.crs.jsj.edu.cn//index.php/default/approval/orglists/2), consultado el 5 de abril de 2016.

MOE (s.f.b): Number of Academic Qualifications of Full-time and Part-time Teachers in HEIs (Regular HEIs) (http://www.moe.gov.cn/s78/A03/moe_560/jytjsj_2014/2014_qg/201509/t20150909 _206892.htm), consultado el 4 de abril de 2016.

MOE (s.f.c): Outline of China's the Eleventh Five-Year Plan of the Development of National

Education 
(http://www.moe.gov.cn/jyb_xwfb/gzdt_gzdt/moe_1485/tnull_22875.html), consultado el 5 de abril de 2016.

NATIONAL BUREAU OF STATISTICS OF THE PEOPLE'S REPUBLIC OF CHINA (2015): National Data (http://data.stats.gov.cn/easyquery.htm?cn=C01\&zb=A0201\&sj=2014)), consultado el 2 de mayo de 2016.

OECD (2004): Internationalisation and Trade in Higher Education: Opportunities and Challenges (París, OECD).

ORR, D. W. (1999): Education for Globalisation, The Ecologist, 29, pp. 166-168.

OTTEN, M. (2000): Impacts of Cultural Diversity at Home, Internationalisation at Home: A Position Paper, pp. 15-20.

PARSONS, L. R. (2010): The effects of an internationalized university experience on domestic students in the United States and Australia, Journal of Studies in International Education, 14, pp. 313-334.

QUACQUARELLI SYMONDS (2016): QS World University Rankings (http://www.topuniversities.com/university-rankings), consultado el 8 de septiembre de 2016.

RAFFE, D. (2011): Policy borrowing or policy learning? How (not) to improve education systems, CES Briefings, 57, pp. 1-4.

SHANGHAI JIAO TONG UNIVERSITY (2015): Academic Ranking of World Universities (http://www.shanghairanking.com/), consultado el 8 de septiembre de 2016.

SHARMA, N. K. (2012): Globalization Effect on Education and Culture: An Analysis (http://ssrn.com/abstract=2069155), consultado el 20 de abril de 2016.

TEICHLER, U. (2004): The Changing Debate on Internationalisation of Higher Education, Higher Education, 48, pp. 5-26.

TIMES HIGHER EDUCATION (2016): World University Rankings (https://www.timeshighereducation.com/), consultado el 8 de septiembre de 2016.

VÁZQUEZ COVARRUBIAS, M. E. (2006): Internacionalización de la educación superior, Revista Vasconcelos De Educación, 2, pp. 88-93.

WÄCHTER, B. (2003): An Introduction: Internationalization at Home in the Context, Journal of Studies in International Education, 7, pp. 5-11.

WANG, F. y SONG, Z. (2003): The development course of the modern and contemporary higher education in China, Journal of Shenyang Normal University (Social Science Edition), 27, pp. 46-48.

WANG, H., ALTYNBEKOVA, D. y LIU, H. (2014): Mobility of International Students and Chinese Policy Choice in the Context of Globalization, Journal of Xiamen University (Arts \& Social Sciences), 2, pp. 149-156.

WANG, L. y CHEN, C. (2007): Construction of Evaluation Index System of Internationalization of Higher Education, Social Sciences in Hubei, 1, pp. 177-180.

XU, J. (2015): The impact of "Internationalization at home" project on the undergraduate students in a Chinese research university: A cross-cultural perspective, Renmin University of China Educational Journal, 3, pp. 143-157.

YUEH, L. (2012): China's 'Going Out, Bringing In' Policy: the Geo-economics of China's rise, IISS Geo-economics and Strategy Programme Seminar, "A New Era of Geoeconomics: Assessing the Interplay of Economic and Political Risk", pp. 23-25, 
Marzo, 2012 (http://www.iiss.org/en/events/geo-economics\%20seminars/geoeconomics\%20seminars/archive/2012-4152/a-new-era-of-geo-economics-

$617 \mathrm{~d} /$ chinas-going-out-bringing-in-policy-the-geo-economics-of-chinas-rise-a541), consultado el 17 de mayo de 2016.

ZHANG, Y. (2012): The Evaluation Index System of Internationalization of Universities, China Higher Education Evaluation, 1, pp. 13-18.

ZHA, Q. (2008): La influencia extranjera en la educación superior de Japón y China: Un análisis comparativo. Revista de la Educación Superior, 145, pp. 125-135.

ZHAO, P. (2014): Ideas on the Internationalization of China's Higher Education, China Education of Light Industry, 5, pp. 19-28.

\section{PROFESIOGRAFÍA}

\section{Xinran Liao}

Universidad Complutense de Madrid. Datos de contacto: Email: vermouth928@163.com

\section{Inmaculada Egido Gálvez}

Doctora en Pedagogía y Catedrática acreditada en el Departamento de Teoría e Historia de la Educación de la Universidad Complutense de Madrid, donde desarrolla su labor docente en el ámbito de la Educación Comparada. Datos de contacto: Facultad de Educación. Universidad Complutense de Madrid. C/ Rector Royo Villanova s/n. 28040 Madrid. Telf.: 913946205. Email: miegido@ucm.es

Fecha de recepción: 24 de mayo de 2016.

Fecha de aceptación: 20 de septiembre de 2016. 